\title{
New approach for the existence and uniqueness of periodic solutions to $p$-Laplacian prescribed mean curvature equations
}

\section{Bo $\mathrm{Du}^{1 *}$ and Weigao $\mathrm{Ge}^{2}$}

\section{"Correspondence:}

dubo7307@163.com

'Department of Mathematics,

Huaiyin Normal University, Huaian,

Jiangsu 223300, P.R. China

Full list of author information is

available at the end of the article

\begin{abstract}
Using a generalized Mawhin continuation theorem, we obtain some sufficient conditions which guarantee the existence and uniqueness of periodic solutions for two types of prescribed mean curvature $p$-Laplacian equations.
\end{abstract}

MSC: 34C57; 58E05

Keywords: generalized Mawhin's continuation theorem; periodic solution; p-Laplacian

\section{Introduction}

Periodic phenomena are omnipresent in the real world. Since Hale [1] first put forward the concept of functional differential equation (FDE), a large amount of results for periodic solutions of FDEs have been obtained; see e.g. [2-9].

In past decades, many authors investigated the existence of periodic solutions for $p$ Laplacian differential equations by using Mawhin's continuation theorem, for more details, see e.g. [10-15] for work on this subject. Now we summarize their research methods. If we set $L=\left(\varphi_{p}\left(x^{\prime}(t)\right)\right)^{\prime}$, then Mawhin's continuation theorem cannot be used directly, because the operator $L$ in Mawhin's continuation theorem need be linear, while $\left(\varphi_{p}\left(x^{\prime}(t)\right)\right)^{\prime}$ is non-linear when $p \neq 2$. In order to use Mawhin's continuation theorem, hence, we must change the form of the source equation. For example, Cheung and Ren [10] considered the following Liénard equation with $p$-Laplacian:

$$
\left(\varphi_{p}\left(u^{\prime}(t)\right)\right)^{\prime}+f(t, u(t)) u^{\prime}(t)+\beta(t) g(u(t-\tau(t)))=e(t) .
$$

Transfer (1.1) into the form

$$
\left\{\begin{array}{l}
u_{1}^{\prime}(t)=\varphi_{q}\left(u_{2}(t)\right) \\
u_{2}^{\prime}(t)=-f\left(t, u_{1}(t)\right) \varphi_{q}\left(u_{2}(t)\right)-\beta(t) g\left(u_{1}(t-\tau(t))\right)+e(t) .
\end{array}\right.
$$

In the same way, in [11], one again investigated $p$-Laplacian Rayleigh equation as follows:

$$
\left(\varphi_{p}\left(u^{\prime}(t)\right)\right)^{\prime}+f\left(t, u^{\prime}(t)\right)+\beta g(t, u(t-\tau(t)))=e(t) .
$$

(c) 2016 Du and Ge. This article is distributed under the terms of the Creative Commons Attribution 4.0 International License (http://creativecommons.org/licenses/by/4.0/), which permits unrestricted use, distribution, and reproduction in any medium, provided you give appropriate credit to the original author(s) and the source, provide a link to the Creative Commons license, and indicate if changes were made. 
Transfer (1.2) into the form

$$
\left\{\begin{array}{l}
u_{1}^{\prime}(t)=\varphi_{q}\left(u_{2}(t)\right), \\
u_{2}^{\prime}(t)=-f\left(\varphi_{q}\left(u_{2}(t)\right)\right)-\beta g\left(u_{1}(t-\tau(t))\right)+e(t) .
\end{array}\right.
$$

Very recently, Wang [16] considered a class of prescribed mean curvature $p$-Laplacian Rayleigh equation with one deviating argument as follows:

$$
\left(\varphi_{p}\left(\frac{x^{\prime}(t)}{\sqrt{1+x^{\prime 2}(t)}}\right)\right)^{\prime}+f\left(x^{\prime}(t)\right)+g(x(t-\tau(t)))=e(t) .
$$

After that, based on the work of [16], Li, An, and Ge [17] studied a class of prescribed mean curvature $p$-Laplacian Liénard equation with two deviating arguments,

$$
\left(\varphi_{p}\left(\frac{x^{\prime}(t)}{\sqrt{1+x^{\prime 2}(t)}}\right)\right)^{\prime}+f(x(t)) x^{\prime}(t)+g(x(t-\tau(t)))+h(x(t-\gamma(t)))=e(t) .
$$

Similar to the above approach, (1.3) and (1.4) can be changed to a two-dimensional system. In addition, we find that the above method can be applied to studying the existence of periodic solutions to neutral equations with $p$-Laplacian, Zhu and Lu [7] considered the following neutral equation with $p$-Laplacian:

$$
\left(\varphi_{p}\left[(u(t)-c u(t-\sigma))^{\prime}\right]\right)^{\prime}+g(t, u(t-\tau(t)))=e(t) .
$$

Transfer (1.5) into the form

$$
\left\{\begin{array}{l}
\left(A u_{1}\right)^{\prime}(t)=\varphi_{q}\left(u_{2}(t)\right), \\
u_{2}^{\prime}(t)=-g\left(t, u_{1}(t-\tau(t))\right)+e(t),
\end{array}\right.
$$

where $A u(t)=u(t)-c u(t-\sigma)$.

In short, most of the papers used this method (transfer the source equation to a twodimensional system) for studying $p$-Laplacian equations. In the face of such a fact, we cannot but ask 'how can we find the different ways for studying the $p$-Laplacian equations?' In order to investigate BVPs with a $p$-Laplacian, Ge and Ren [18] extended Mawhin's continuation theorem. The above generalized theorem removes the restriction that $L$ is a linear operator. Motivated by the work of Ge and Ren, in the present paper we will study the existence and uniqueness of periodic solutions for equations (1.6) and (1.7) by using the generalized Mawhin's continuation theorem. Here, it is worth stating that the forms of equations (1.6) and (1.7) need not to be transferred to a two-dimensional system. The major challenges are as follows: (1) in order to apply a generalized Mawhin's continuation theorem, the proof of $M$-compactness (see Definition 2.2) of the operators is very difficult; (2) equations (1.6) and (1.7) are of much stronger non-linearity, so how to estimate the prior bounds for periodic solutions is crucial; and (3) it is non-trivial to establish a unified framework to handle the prescribed mean curvature term and the influence of variable delays. It is, therefore, the main purpose of this paper to make the first attempt to handle the listed challenges.

On the other hand, the dynamic properties of Duffing and Rayleigh equations have been widely investigated due to the applications in many fields, such as mechanics, physics, 
and the engineering technique fields; see e.g. [19-22]. In such applications, it is important to know the existence and uniqueness of periodic solution to the Rayleigh and Duffing equations. We note that $p$-Laplacian equations have been widely applied in the field of non-linear elastic mechanics and fluid mechanics, it is necessary to study the periodic solutions of FDEs involving the scalar $p$-Laplacian. For the problems of periodic solutions for $p$-Laplacian equations, see e.g. [7, 8, 10-13]. This paper is devoted to an investigation of the existence and uniqueness of periodic solutions for a prescribed mean curvature $p$-Laplacian Rayleigh equation and a $p$-Laplacian Duffing equation as follows:

$$
\left(\varphi_{p}\left(\frac{x^{\prime}(t)}{\sqrt{1+x^{\prime 2}(t)}}\right)\right)^{\prime}+f\left(x^{\prime}(t)\right)+g\left(x\left(t-\tau_{1}(t)\right)\right)=e_{1}(t)
$$

and

$$
\left(\varphi_{p}\left(\frac{x^{\prime}(t)}{\sqrt{1+x^{\prime 2}(t)}}\right)\right)^{\prime}+g\left(x\left(t-\tau_{2}(t)\right)\right)=e_{2}(t)
$$

where $\varphi_{p}(s)=|s|^{p-2} s, p>1, \varphi_{q}=\varphi_{p}^{-1}, \frac{1}{p}+\frac{1}{q}=1 ; f, g, e_{i}, \tau_{i} \in C(\mathbb{R}, \mathbb{R})$ where $e_{i}(t)=e_{i}(t+T)$ and $\tau_{i}(t)=\tau_{i}(t+T), i=1,2, T>0$, is a given constant.

Remark 1.1 Equations (1.6) and (1.7) are the one-dimensional version of the elliptic Dirichlet problem,

$$
-\operatorname{div}\left(\frac{\nabla x}{\sqrt{1+\|\nabla x\|^{2}}}\right)=F(t, x) \text { in } \Omega, x=0 \text { on } \partial \Omega \text {. }
$$

It is well known that a solution $x$ of (1.8) defines a Cartesian in $\mathbb{R}^{N+1}$ whose mean curvature is prescribed by the right-hand side of the equation. Such problems have attracted much attention by many researches; see e.g. [23-27] and the references therein.

Remark 1.2 As for equations (1.6) and (1.7), a common method for study is to transfer the forms of equations (1.6) and (1.7) into the forms as follows, respectively:

$$
\left\{\begin{array}{l}
x_{1}^{\prime}(t)=\frac{\varphi_{q}\left(x_{2}(t)\right)}{\sqrt{1-\varphi_{q}^{2}\left(x_{2}(t)\right)}}, \\
x_{2}^{\prime}(t)=-f\left(\frac{\varphi_{q}\left(x_{2}(t)\right)}{\sqrt{1-\varphi_{q}^{2}\left(x_{2}(t)\right)}}\right)-g\left(x_{1}\left(t-\tau_{1}(t)\right)\right)+e_{1}(t),
\end{array}\right.
$$

and

$$
\left\{\begin{array}{l}
x_{1}^{\prime}(t)=\varphi_{q}\left(\frac{\varphi_{q}\left(x_{2}(t)\right)}{\sqrt{1-\varphi_{q}^{2}\left(x_{2}(t)\right)}}\right), \\
x_{2}^{\prime}(t)=-g\left(x_{1}\left(t-\tau_{2}(t)\right)\right)+e_{2}(t) .
\end{array}\right.
$$

Let $L=\left(x_{1}^{\prime}, x_{2}^{\prime}\right)^{T}$, then $L$ is a linear operator. Thus Mawhin's continuation theorem can be used to study the existence and uniqueness of periodic solutions to equation (1.9) and equation (1.10).

To the best of our knowledge, there are few results for studying the existence and uniqueness of periodic solutions to $p$-Laplacian prescribed mean curvature equations by using 
the generalized Mawhin continuation theorem, the main purpose of this paper is to introduce a new method for studying the above equations. As to methods for studying the existence and uniqueness of periodic solutions, such as the Lyapunov second method, fixed point theorem, coincidence degree theory, the Yoshizawa type theorem, and the Massera type theorem, see $[1,28-30]$ and references therein.

The following sections are organized as follows: In Section 2, we give some useful lemmas and definitions. In Section 3, sufficient conditions are established for existence results of periodic solutions to equations (1.6) and (1.7). In Section 4, sufficient conditions are established for uniqueness results of periodic solutions to equations (1.6) and (1.7). In Section 5, some examples are given to show the feasibility of our results. Finally, some conclusions are given for this paper.

\section{Preliminary}

In this section, we give some definitions and lemmas which will be used in this paper.

Definition 2.1 ([18]) Denote $X$ and $Z$ two Banach spaces with norms $\|\cdot\|_{X},\|\cdot\|_{Z}$, respectively. A continuous operator

$$
\mathcal{M}: X \cap \operatorname{dom} \mathcal{M} \rightarrow Z
$$

is said to be quasi-linear if

(i) $\operatorname{Im} \mathcal{M}:=\mathcal{M}(X \cap \operatorname{dom} M)$ is a closed subset of $Z$;

(ii) $\operatorname{Ker} \mathcal{M}:=\{x \in X \cap \operatorname{dom} \mathcal{M}: \mathcal{M} x=0\}$ is linearly homeomorphic to $\mathbb{R}^{n}, n<\infty$.

Definition 2.2 ([18]) Let $\Omega \subset X$ be an open and bounded set with the origin $\theta \in \Omega$. $N_{\lambda}$ : $\bar{\Omega} \rightarrow Z, \lambda \in[0,1]$ is said to be $\mathcal{M}$-compact in $\bar{\Omega}$ if there exists a subset $Z_{1}$ of $Z$ satisfying $\operatorname{dim} Z_{1}=\operatorname{dim} \operatorname{Ker} \mathcal{M}$ and an operator $R: \bar{\Omega} \times[0,1] \rightarrow X_{2}$ being continuous and compact such that, for $\lambda \in[0,1]$,

(a) $(I-Q) N_{\lambda}(\bar{\Omega}) \subset \operatorname{Im} \mathcal{M} \subset(I-Q) Z$,

(b) $Q N_{\lambda} x=0, \lambda \in(0,1) \Leftrightarrow Q N x=0, \forall x \in \Omega$,

(c) $R(\cdot, 0) \equiv 0$ and $\left.R(\cdot, \lambda)\right|_{\Sigma_{\lambda}}=\left.(I-P)\right|_{\Sigma_{\lambda}}$

(d) $\mathcal{M}[P+R(\cdot, \lambda)]=(I-Q) N_{\lambda}, \lambda \in[0,1]$,

where $X_{2}$ is a complement space of $\operatorname{Ker} \mathcal{M}$ in $X$, i.e., $X=\operatorname{Ker} \mathcal{M} \oplus X_{2} ; P, Q$ are two projectors satisfying $\operatorname{Im} P=\operatorname{Ker} \mathcal{M}, \operatorname{Im} Q=Z_{1}, N=N_{1}, \Sigma_{\lambda}=\left\{x \in \bar{\Omega}: \mathcal{M} x=N_{\lambda} x\right\}$.

Lemma 2.1 ([18]) Let $X$ and $Z$ be two Banach spaces with norms $\|\cdot\|_{X},\|\cdot\|_{Z}$, respectively, and $\Omega \subset X$ be an open and bounded nonempty set. Suppose

$$
M: X \cap \operatorname{dom} \mathcal{M} \rightarrow Z
$$

is quasi-linear and $N_{\lambda}: \bar{\Omega} \rightarrow Z, \lambda \in[0,1]$ is $M$-compact in $\bar{\Omega}$. In addition, if the following conditions hold:

$\left(\mathrm{H}_{1}\right) \quad \mathcal{M} x \neq N_{\lambda} x, \forall(x, \lambda) \in \partial \Omega \times(0,1)$;

$\left(\mathrm{H}_{2}\right) \quad Q N x \neq 0, \forall x \in \operatorname{Ker} \mathcal{M} \cap \partial \Omega$;

$\left(\mathrm{H}_{3}\right) \operatorname{deg}\{J Q N, \Omega \cap \operatorname{Ker} \mathcal{M}, 0\} \neq 0, J: \operatorname{Im} Q \rightarrow \operatorname{Ker} \mathcal{M}$ is a homeomorphism,

then the abstract equation $\mathcal{M} x=N x$ has at least one solution in $\operatorname{dom} \mathcal{M} \cap \bar{\Omega}$. 
Lemma 2.2 ([12]) Let $p \in(1,+\infty)$ be a constant, $s \in C(\mathbb{R}, \mathbb{R})$ such that $s(t+T) \equiv s(t)$, $u \in C^{1}(R, R)$ with $u(t+T) \equiv u(t)$. Then

$$
\int_{0}^{T}|u(t)-u(t-s(t))|^{p} d t \leq 2\left(\max _{t \in[0, T]}|s(t)|\right)^{p} \int_{0}^{T}\left|u^{\prime}(t)\right|^{p} d t .
$$

Throughout this paper, assume that $e_{i}(t)(i=1,2)$ is not a constant function on $\mathbb{R}$. Furthermore, we suppose that $\tau_{i} \in C^{1}(\mathbb{R}, \mathbb{R})$ with $\tau_{i}^{\prime}(t)<1, \forall t \in \mathbb{R}, i=1,2$. It is clear that the function $t-\tau_{i}(t)$ has a unique inverse, denoted by $\gamma_{i}(t)$.

Now, we list the following assumptions:

[A1] there is a constant $\sigma \geq 0$ such that

$$
f(u) u \geq \sigma|u|^{p}, \quad \forall u \in \mathbb{R}
$$

or

$$
f(u) u \leq-\sigma|u|^{p}, \quad \forall u \in \mathbb{R}
$$

[A2] there is a constant $\sigma \geq 0$ such that

$$
f(u) \geq \sigma|u|^{p}, \quad \forall u \in \mathbb{R}
$$

or

$$
f(u) \leq-\sigma|u|^{p}, \quad \forall u \in \mathbb{R} ;
$$

[A3] there is a constant $l \geq 0$ such that

$$
\left|g\left(v_{1}\right)-g\left(v_{2}\right)\right| \leq l\left|v_{1}-v_{2}\right|, \quad \forall v_{1}, v_{2} \in \mathbb{R} ;
$$

[A4] there exists a constant $r \geq 0$ such that

$$
\lim _{|u| \rightarrow+\infty} \frac{|g(u)|}{|u|^{m}} \leq r, \quad m \in \mathbb{N} ;
$$

[A5] there is a constant $D>0$ such that

$$
g(x)<-|e|_{0}-|f(0)| \text { for } x>D
$$

and

$$
g(x)>|e|_{0}+|f(0)| \quad \text { for } x<-D
$$

[A6] there is a constant $D_{1}>0$ such that

$$
g(x)<-|e|_{0} \quad \text { for } x>D_{1}
$$


and

$g(x)>|e|_{0} \quad$ for $x<-D_{1}$

[A7] there is a constant $\sigma \geq 0$ such that

$g(x) \geq \sigma|x|^{m}, \quad \forall x \in \mathbb{R}, m \in \mathbb{N}$

or

$g(x) \leq-\sigma|x|^{m}, \quad \forall x \in \mathbb{R}, m \in \mathbb{N}$.

\section{Existence of periodic solutions}

Denote $C_{T}=\{u \mid u \in C(\mathbb{R}, \mathbb{R}), u(t+T)=u(t)\}, C_{T}^{1}=\left\{u \mid u \in C^{1}(\mathbb{R}, \mathbb{R}), u(t+T)=u(t)\right\}, X=$ $C_{T}^{1}$ with the norm $\|u\|=\max \left\{|u|_{0},\left|u^{\prime}\right|_{0}\right\}$, where $|u|_{0}=\max _{0 \leq t \leq T}|u(t)|, Z=C_{T}$, then the operators $\mathcal{M}, N_{\lambda}$ are defined by

$$
\begin{aligned}
& \mathcal{M}: \operatorname{dom} \mathcal{M} \cap X \rightarrow Z, \quad(\mathcal{M} x)(t)=\left(\varphi_{p}\left(\frac{x^{\prime}(t)}{\sqrt{1+x^{\prime 2}(t)}}\right)\right)^{\prime}, \quad t \in \mathbb{R}, \\
& N_{\lambda}: X \rightarrow Z, \quad\left(N_{\lambda} x\right)(t)=-\lambda f\left(x^{\prime}(t)\right)-\lambda g\left(x\left(t-\tau_{1}(t)\right)\right)+\lambda e_{1}(t), \\
& t \in \mathbb{R}, \lambda \in[0,1],
\end{aligned}
$$

where $\operatorname{dom} \mathcal{M}=\left\{x \in X: \varphi_{p}\left(x^{\prime}(t) / \sqrt{1+x^{\prime 2}(t)}\right) \in C_{T}^{1}\right\}$. Denote

$$
\mathcal{F}\left(t, x(t), x^{\prime}(t), x\left(t-\tau_{1}(t)\right)\right)=-f\left(x^{\prime}(t)\right)-g\left(x\left(t-\tau_{1}(t)\right)\right)+e_{1}(t)
$$

then $\left(N_{\lambda} x\right)(t)=\lambda F$. By (3.1) and (3.2), equation (1.6) is equivalent to the operator equation $N x=\mathcal{M} x$, where $N_{1}=N$. Then we have

$$
\begin{aligned}
& \operatorname{Ker} \mathcal{M}=\{x \in \operatorname{dom} \mathcal{M} \cap X: x(t)=a, a \in \mathbb{R}, t \in \mathbb{R}\} \\
& \operatorname{Im} \mathcal{M}=\left\{z \in Z: \int_{0}^{T} z(s) d s=0\right\} .
\end{aligned}
$$

Clearly, $\operatorname{Ker} M \cong \mathbb{R}, \operatorname{Im} M$ is a closed set in $Z$, we have

Lemma 3.1 $\mathcal{M}$ is defined by (3.1), then $\mathcal{M}$ is a quasi-linear operator.

Let

$$
\begin{aligned}
& P: X \rightarrow \operatorname{Ker} \mathcal{M}, \quad(P x)(t)=x(0), \quad t \in \mathbb{R}, \\
& Q: Z \rightarrow \mathbb{R}, \quad(Q z)(t)=\frac{1}{T} \int_{0}^{T} z(s) d s
\end{aligned}
$$

Lemma 3.2 If $f, g, e_{1}, \tau_{1} \in C(\mathbb{R}, \mathbb{R})$ with $e_{1}(t)=e_{1}(t+T)$ and $\tau_{1}(t)=\tau_{1}(t+T)$, then $N_{\lambda}$ is M-compact. 
Proof Denote $Z_{1}=\operatorname{Im} Q$. For each bounded set $\bar{\Omega} \subset X \neq \emptyset, \forall x \in \bar{\Omega}$, since $x(0)=x(T)$, there is a point $\eta \in[0, T]$ such that

$$
x^{\prime}(\eta)=0 .
$$

Define the operator: $R: \bar{\Omega} \times[0,1] \rightarrow \operatorname{Ker} P$,

$$
\begin{aligned}
R(x, \lambda)(t)= & \int_{0}^{t} \sqrt{1+x^{\prime 2}(s)} \varphi_{q} \\
& \times\left[\int_{\eta}^{s} \lambda\left(\mathcal{F}\left(r, x(r), x^{\prime}(r), x(r-\tau(r))\right)-(Q \mathcal{F})(r)\right) d r\right] d s, \quad t \in[0, T],
\end{aligned}
$$

where $\mathcal{F}$ is defined by (3.3), $\eta$ is defined by (3.4). Based on the properties of $f, g, \tau_{1}, e_{1}$, we see that $R(\cdot, \lambda)$ is continuous and relatively compact. Then we show that $N_{\lambda}$ is $\mathcal{M}$-compact in four steps.

Step 1. Using $Q^{2}=Q$, we get $Q(I-Q) N_{\lambda}(\bar{\Omega})=0$, so $(I-Q) N_{\lambda}(\bar{\Omega}) \subset \operatorname{Ker} Q=\operatorname{Im} \mathcal{M}$. On the other hand, $\forall z \in \operatorname{Im} \mathcal{M}$, obviously $Q z=0$, so $z=z-Q z=(I-Q) z$, then $z \in(I-Q) Z$. Thus,

$$
(I-Q) N_{\lambda}(\bar{\Omega}) \subset \operatorname{Im} \mathcal{M} \subset(I-Q) Z .
$$

Step 2. We verify that $Q N_{\lambda} x=0, \lambda \in(0,1) \Leftrightarrow Q N x=0, \forall x \in \Omega$. Since $Q N_{\lambda} x=\frac{1}{T} \int \lambda \mathcal{F} d r=$ 0 , then $\frac{1}{T} \int \mathcal{F} d r=0$, i.e., $Q N x=0$. The inverse is true.

Step 3. Obviously $R(\cdot, 0)=0 . \forall x \in \Sigma_{\lambda}=\left\{x \in \bar{\Omega}: \mathcal{M} x=N_{\lambda} x\right\}$, we have $\left(\varphi_{p}\left(x^{\prime} / \sqrt{1+x^{\prime 2}}\right)\right)^{\prime}=$ $\lambda \mathcal{F}$ and $Q \mathcal{F}=0$. Hence

$$
\begin{aligned}
R(x, \lambda)(t) & =\int_{0}^{t} \sqrt{1+x^{\prime 2}(s)} \varphi_{q}\left[\int_{\eta}^{s} \lambda\left(\mathcal{F}\left(r, x(r), x^{\prime}(r), x(r-\tau(r))\right)-(Q \mathcal{F})(r)\right) d r\right] d s \\
& =\int_{0}^{t} \sqrt{1+x^{\prime 2}(s)} \varphi_{q}\left[\int_{\eta}^{s} \lambda \mathcal{F}\left(r, x(r), x^{\prime}(r), x(r-\tau(r))\right) d r\right] d s \\
& =\int_{0}^{t} \sqrt{1+x^{\prime 2}(s)} \varphi_{q}\left[\int_{\eta}^{s}\left(\varphi_{p}\left(x^{\prime} / \sqrt{1+x^{\prime 2}(r)}\right)\right)^{\prime} d r\right] d s \\
& =\int_{0}^{t} x^{\prime}(s) d s \\
& =x(t)-x(0) \\
& =[(I-P) x](t) .
\end{aligned}
$$

Step 4. $\forall x \in \bar{\Omega}$, we get

$$
\begin{aligned}
\mathcal{M} & P x+R(x, \lambda)](t) \\
= & \left(\varphi _ { p } \left(\left[x(0)+\int_{0}^{t} \sqrt{1+x^{\prime 2}(s)} \varphi_{q}\left[\int _ { \eta } ^ { s } \lambda \left(\mathcal{F}\left(r, x(r), x^{\prime}(r), x(r-\tau(r))\right)\right.\right.\right.\right.\right. \\
& \left.\left.-(Q \mathcal{F})(r)) d r] d s]^{\prime}\right)\right)^{\prime} \\
= & {\left[(I-Q) N_{\lambda} x\right](t) . }
\end{aligned}
$$

Hence, $N_{\lambda}$ is $\mathcal{M}$-compact in $\bar{\Omega}$. 
Theorem 3.1 Assume that assumptions [A1], [A3], and [A5] hold, then equation (1.6) has at least one T-periodic solution.

Proof We will complete the proof in three steps.

Step 1 . Let $\Omega_{1}=\left\{x \in \operatorname{dom} M: \mathcal{M} x=N_{\lambda} x, \lambda \in(0,1)\right\}$. We verify that $\Omega_{1}$ is a bounded set. If $x \in \Omega_{1}$, then $\mathcal{M} x=N_{\lambda} x$, i.e.,

$$
\left(\varphi_{p}\left(\frac{x^{\prime}(t)}{\sqrt{1+x^{\prime 2}(t)}}\right)\right)^{\prime}=-\lambda f\left(x^{\prime}(t)\right)-\lambda g\left(x\left(t-\tau_{1}(t)\right)\right)+\lambda e_{1}(t)
$$

Let $t_{0}$ be the maximum point of $x(t)$ on $[0, T]$, i.e., $x\left(t_{0}\right)=\max _{t \in[0, T]} x(t)$. Then $x^{\prime}\left(t_{0}\right)=0$, by (3.5), we get

$$
g\left(x\left(t_{0}-\tau_{1}\left(t_{0}\right)\right)\right)=-f(0)+e_{1}\left(t_{0}\right) \geq-|f(0)|-\left|e_{1}\right|_{0}
$$

and

$$
g\left(x\left(t_{0}-\tau_{1}\left(t_{0}\right)\right)\right) \leq|f(0)|+\left|e_{1}\right|_{0}
$$

which together with the condition [A5] shows that we have $-D \leq x\left(t_{0}-\tau_{1}\left(t_{0}\right)\right) \leq D$. Since $x(t)$ is a $T$-periodic function, there is a constant $\xi^{*} \in[0, T]$ satisfying $t_{0}-\tau_{1}\left(t_{0}\right)=\xi^{*}+$ $k T, k \in \mathbb{Z}$, and we get

$$
\left|x\left(\xi^{*}\right)\right| \leq D
$$

By (3.6) we have

$$
|x|_{0}=\max _{t \in[0, T]}\left|x\left(\xi^{*}\right)+\int_{\xi^{*}}^{t} x^{\prime}(s) d s\right| \leq\left|x\left(\xi^{*}\right)\right|+\int_{0}^{T}\left|x^{\prime}(s)\right| d s \leq D+\int_{0}^{T}\left|x^{\prime}(s)\right| d s .
$$

Multiplying two sides of equation (3.5) by $x^{\prime}(t)$ and integrating over $[0, T]$, we get

$$
\begin{aligned}
& \int_{0}^{T}\left(\varphi_{p}\left(\frac{x^{\prime}(t)}{\sqrt{1+x^{\prime 2}(t)}}\right)\right)^{\prime} x^{\prime}(t) d t+\lambda \int_{0}^{T} f\left(x^{\prime}(t)\right) x^{\prime}(t) d t+\lambda \int_{0}^{T} g\left(x\left(t-\tau_{1}(t)\right)\right) x^{\prime}(t) d t \\
& \quad=\lambda \int_{0}^{T} e_{1}(t) x^{\prime}(t) d t
\end{aligned}
$$

Denote $\omega(t)=\varphi_{p}\left(\frac{x^{\prime}(t)}{\sqrt{1+x^{\prime 2}(t)}}\right)$, then $x^{\prime}(t)= \pm \sqrt{\frac{\varphi_{q}^{2}(\omega(t))}{1-\varphi_{q}^{2}(\omega(t))}}$ and

$$
\int_{0}^{T}\left(\varphi_{p}\left(\frac{x^{\prime}(t)}{\sqrt{1+x^{\prime 2}(t)}}\right)\right)^{\prime} x^{\prime}(t) d t= \pm \int_{0}^{T} \sqrt{\frac{\varphi_{q}^{2}(\omega(t))}{1-\varphi_{q}^{2}(\omega(t))}} d \omega(t)=0 .
$$

Hence equation (3.8) reduces to

$$
\int_{0}^{T} f\left(x^{\prime}(t)\right) x^{\prime}(t) d t=-\int_{0}^{T} g\left(x\left(t-\tau_{1}(t)\right)\right) x^{\prime}(t) d t+\int_{0}^{T} e_{1}(t) x^{\prime}(t) d t
$$


From condition [A1], we have

$$
\left|\int_{0}^{T} f\left(x^{\prime}(t)\right) x^{\prime}(t) d t\right|=\int_{0}^{T}\left|f\left(x^{\prime}(t)\right) x^{\prime}(t)\right| d t \geq \sigma \int_{0}^{T}\left|x^{\prime}(t)\right|^{p} d t
$$

which together with [A3], Lemma 2.2, (3.9), and the Hölder inequality implies

$$
\begin{aligned}
\sigma \int_{0}^{T}\left|x^{\prime}(t)\right|^{p} d t \leq & \int_{0}^{T}\left|g\left(x\left(t-\tau_{1}(t)\right)\right) x^{\prime}(t)\right| d t+\int_{0}^{T}\left|e_{1}(t) x^{\prime}(t)\right| d t \\
\leq & \int_{0}^{T}\left|g\left(x\left(t-\tau_{1}(t)\right)\right) x^{\prime}(t)\right| d t+\left\|e_{1}\right\|_{q}\left(\int_{0}^{T}\left|x^{\prime}(t)\right|^{p} d t\right)^{\frac{1}{p}} \\
\leq & \int_{0}^{T}\left|\left[g\left(x\left(t-\tau_{1}(t)\right)\right)-g(x(t))\right] x^{\prime}(t)\right| d t \\
& +\int_{0}^{T}\left|g(x(t)) x^{\prime}(t)\right| d t+\left\|e_{1}\right\|_{q}\left(\int_{0}^{T}\left|x^{\prime}(t)\right|^{p} d t\right)^{\frac{1}{p}} \\
\leq & \int_{0}^{T} l\left|\left[x\left(t-\tau_{1}(t)\right)-x(t)\right] x^{\prime}(t)\right| d t+\left\|e_{1}\right\|_{q}\left(\int_{0}^{T}\left|x^{\prime}(t)\right|^{p} d t\right)^{\frac{1}{p}} \\
\leq & l\left(\int_{0}^{T}\left|x\left(t-\tau_{1}(t)\right)-x(t)\right|^{q}\right)^{1 / q}\left(\int_{0}^{T}\left|x^{\prime}(t)\right|^{p} d t\right)^{1 / p} \\
& +\left\|e_{1}\right\|_{q}\left(\int_{0}^{T}\left|x^{\prime}(t)\right|^{p} d t\right)^{\frac{1}{p}} \\
\leq & l 2^{1 / q}\left|\tau_{1}\right|_{0}\left(\int_{0}^{T}\left|x^{\prime}(t)\right|^{q}\right)^{1 / q}\left(\int_{0}^{T}\left|x^{\prime}(t)\right|^{p} d t\right)^{1 / p} \\
& +\left\|e_{1}\right\|_{q}\left(\int_{0}^{T}\left|x^{\prime}(t)\right|^{p} d t\right)^{\frac{1}{p}} .
\end{aligned}
$$

By $p, q>1$, and (3.10), there must be a constant $M_{1}>0$ such that

$$
\int_{0}^{T}\left|x^{\prime}(t)\right|^{p} d t \leq M_{1}
$$

Thus by the Hölder inequality and (3.7), we have

$$
|x|_{0} \leq D+T^{1 / q}\left(\int_{0}^{T}\left|x^{\prime}(t)\right|^{p} d t\right)^{1 / p} \leq D+T^{1 / q} M_{1}^{1 / p}:=M_{2}
$$

By (3.11), we have $\left|x^{\prime}\right|_{0} \leq T^{-\frac{1}{p}} M_{1}^{\frac{1}{p}}:=M_{3}$. Thus,

$$
\|x\|<\max \left\{M_{2}, M_{3}\right\}+1:=\tilde{M} .
$$

Step 2. Let $\Omega_{2}=\{x \in \operatorname{Ker} \mathcal{M}: Q N x=0\}$. We prove that $\Omega_{2}$ is a bounded set. $\forall x \in \Omega_{2}$, then $x=a_{0}, a_{0} \in \mathbb{R}$, and we have

$$
\int_{0}^{T}\left(g\left(a_{0}\right)+f(0)-e_{1}(t)\right) d t=0 .
$$


Based on the integral mean value theorem, there exists a constant $t_{1} \in[0, T]$ such that

$$
g\left(a_{0}\right)+f(0)-e_{1}\left(t_{1}\right)=0,
$$

by [A5], we have $\left|a_{0}\right| \leq D$. So $\Omega_{2}$ is a bounded set.

Step 3. Let $\Omega=\{x \in X:\|x\|<\widetilde{M}\}$, then $\Omega_{1} \cup \Omega_{2} \subset \Omega . \forall(x, \lambda) \in \partial \Omega \times(0,1)$, from the above proof, $\mathcal{M} x \neq N_{\lambda} x$ is satisfied. Clearly, condition $\left(\mathrm{H}_{2}\right)$ of Lemma 2.1 is satisfied. Now, we show that condition $\left(\mathrm{H}_{3}\right)$ of Lemma 2.1 holds. Take the homotopy

$$
H(x, \mu)=-\mu x+(1-\mu) J Q N x, \quad x \in \bar{\Omega} \cap \operatorname{Ker} \mathcal{M}, \mu \in[0,1],
$$

where $J: \operatorname{Im} Q \rightarrow \operatorname{Ker} M$ is a homeomorphism with $J a=a, a \in \mathbb{R} . \forall x \in \partial \Omega \cap \operatorname{Ker} M$, we have $x=a_{1},\left|a_{1}\right|=\widetilde{M}>D$, then

$$
\begin{aligned}
& H(x, \mu)=-a_{1} \mu+(1-\mu) \frac{1}{T} \int_{0}^{T}\left(-g\left(a_{1}\right)-f(0)+e_{1}(t)\right) d t, \\
& a_{1} H(x, \mu)=-a_{1}^{2} \mu+(1-\mu) \frac{1}{T} \int_{0}^{T} a_{1}\left(-g\left(a_{1}\right)-f(0)+e_{1}(t)\right) d t .
\end{aligned}
$$

By assumption [A5], we have $H(x, \mu)<0$. Using degree theory, we have

$$
\begin{aligned}
\operatorname{deg}\{J Q N, \Omega \cap \operatorname{Ker} \mathcal{M}, 0\} & =\operatorname{deg}\{H(\cdot, 0), \Omega \cap \operatorname{Ker} \mathcal{M}, 0\} \\
& =\operatorname{deg}\{H(\cdot, 1), \Omega \cap \operatorname{Ker} \mathcal{M}, 0\} \\
& =\operatorname{deg}\{-I, \Omega \cap \operatorname{Ker} \mathcal{M}, 0\} \neq 0 .
\end{aligned}
$$

Using Lemma 2.1, we reach the conclusion.

Remark 3.1 Assumptions [A1] and [A2] ensure that integrals $\int_{0}^{T} f(u(t)) u(t) d t$ and $\int_{0}^{T} f(u(t)) d t$ have no sign change, respectively.

Remark 3.2 Assumption [A5] is crucial for obtaining prior bounds of periodic solutions and verifying the second condition of Lemma 2.1 .

Theorem 3.2 Suppose that assumptions [A2], [A4], and [A5] hold, then equation (1.6) has at least one T-periodic solution if

(1) $m=p, \sigma>\max _{t \in[0, T]}\left|\frac{1}{1-\tau_{1}^{\prime}\left(\gamma_{1}(t)\right)}\right| r$, or

(2) $m<p$.

Proof Similar to the proof of Theorem 3.1, integrating the two sides of (3.5) over [0,T], we have

$$
\int_{0}^{T} f\left(x^{\prime}(t)\right) d t+\int_{0}^{T} g\left(x\left(t-\tau_{1}(t)\right)\right) d t=\int_{0}^{T} e_{1}(t) d t
$$

In view of [A2], we have

$$
\left|\int_{0}^{T} f\left(x^{\prime}(t)\right) d t\right|=\int_{0}^{T}\left|f\left(x^{\prime}(t)\right)\right| \geq \sigma \int_{0}^{T}\left|x^{\prime}(t)\right|^{p} d t
$$


and so

$$
\sigma \int_{0}^{T}\left|x^{\prime}(t)\right|^{p} d t \leq \int_{0}^{T}\left|g\left(x\left(t-\tau_{1}(t)\right)\right)\right| d t+\int_{0}^{T}\left|e_{1}(t)\right| d t .
$$

From assumption [A4], $\forall \varepsilon>0$, we see that there is a constant $\rho>D>0$ such that

$$
|g(x)| \leq(r+\varepsilon)|x|^{m}, \quad \text { whenever }|x|>\rho .
$$

Let

$$
E_{1}=\left\{t \in[0, T]:\left|x\left(t-\tau_{1}(t)\right)\right|>\rho\right\}, \quad E_{2}=\left\{t \in[0, T]:\left|x\left(t-\tau_{1}(t)\right)\right| \leq \rho\right\} .
$$

By (3.12) and (3.13), we have

$$
\begin{aligned}
\sigma \int_{0}^{T}\left|x^{\prime}(t)\right|^{p} d t & \leq \int_{E_{1}}\left|g\left(x\left(t-\tau_{1}(t)\right)\right)\right| d t+\int_{E_{2}}\left|g\left(x\left(t-\tau_{1}(t)\right)\right)\right| d t+T\left|e_{1}\right|_{0} \\
& \leq \int_{0}^{T}(r+\varepsilon)\left|x\left(t-\tau_{1}(t)\right)\right|^{m} d t+T g_{\rho}+T\left|e_{1}\right|_{0} \\
& \leq \max _{t \in[0, T]}\left|\frac{1}{1-\tau_{1}^{\prime}\left(\gamma_{1}(t)\right)}\right|(r+\varepsilon) \int_{0}^{T}|x(t)|^{m} d t+T g_{\rho}+T\left|e_{1}\right|_{0}
\end{aligned}
$$

where $g_{\rho}=\max _{|v| \leq \rho}|g(v)|$.

Case 1. If $p=m$ and $\sigma>\max _{t \in[0, T]}\left|\frac{1}{1-\tau_{1}^{\prime}\left(\gamma_{1}(t)\right)}\right| r$. Choose $\varepsilon<\sigma-\max _{t \in[0, T]}\left|\frac{1}{1-\tau_{1}^{\prime}\left(\gamma_{1}(t)\right)}\right| r$. So by (3.14), $\int_{0}^{T}\left|x^{\prime}(t)\right|^{p} d t$ is bounded.

Case 2. If $m<p$, by the Hölder inequality and (3.14), we get

$$
\begin{aligned}
\sigma \int_{0}^{T}\left|x^{\prime}(t)\right|^{p} d t \leq & \max _{t \in[0, T]}\left|\frac{1}{1-\tau_{1}^{\prime}\left(\gamma_{1}(t)\right)}\right|(r+\varepsilon) T^{p-m / p}\left(\int_{0}^{T}|x(t)|^{p} d t\right)^{m / p} \\
& +T g_{\rho}+T\left|e_{1}\right|_{0} .
\end{aligned}
$$

From $m / p<1$ and (3.15), we see that $\int_{0}^{T}\left|x^{\prime}(t)\right|^{p} d t$ is bounded. Hence we know that in two cases there exists a constant $M>0$ such that

$$
\int_{0}^{T}\left|x^{\prime}(t)\right|^{p} d t<M
$$

which together with (3.7) yields

$$
|x|_{0} \leq D+T^{1 / q}\left(\int_{0}^{T}\left|x^{\prime}(t)\right|^{p} d t\right)^{1 / p} \leq D+T^{1 / q} M^{1 / p}
$$

The rest of the proof of the theorem is identical to that of Theorem 3.1.

Remark 3.3 The results of Theorem 3.2 show that the existence of a periodic solution is related to the delays.

Now, we give an existence theorem for equation (1.7). 
Theorem 3.3 Suppose that assumptions [A6] and [A7] hold, then equation (1.7) has at least one T-periodic solution $x$.

Proof Similar to the proof of Theorem 3.1, let

$$
\begin{aligned}
& \mathcal{M}: \operatorname{dom} \mathcal{M} \cap X \rightarrow Z, \quad(\mathcal{M} x)(t)=\left(\varphi_{p}\left(\frac{x^{\prime}(t)}{\sqrt{1+x^{\prime 2}(t)}}\right)\right)^{\prime}, \quad t \in \mathbb{R}, \\
& N_{\lambda}: X \rightarrow Z, \quad\left(N_{\lambda} x\right)(t)=-\lambda g\left(x\left(t-\tau_{2}(t)\right)\right)+\lambda e_{2}(t), \quad t \in \mathbb{R}, \lambda \in[0,1] .
\end{aligned}
$$

Let $\Omega_{1}=\left\{x \in \operatorname{dom} \mathcal{M}: \mathcal{M} x=N_{\lambda} x, \lambda \in(0,1)\right\}$, where $\mathcal{M}$ and $N_{\lambda}$ are defined by (3.16) and (3.17), respectively. We show that $\Omega_{1}$ is a bounded set. If $x \in \Omega_{1}$, then $\mathcal{M} x=N_{\lambda} x$, i.e.,

$$
\left(\varphi_{p}\left(\frac{x^{\prime}(t)}{\sqrt{1+x^{\prime 2}(t)}}\right)\right)^{\prime}=-\lambda g\left(x\left(t-\tau_{2}(t)\right)\right)+\lambda e_{2}(t) .
$$

Similar to (3.6) and (3.7), by assumption [A6] there exists a constant $\eta^{*} \in[0, T]$ such that

$$
\left|x\left(\eta^{*}\right)\right| \leq D_{1}
$$

and

$$
|x|_{0}=\max _{t \in[0, T]}\left|x\left(\eta^{*}\right)+\int_{\eta^{*}}^{t} x^{\prime}(s) d s\right| \leq\left|x\left(\eta^{*}\right)\right|+\int_{0}^{T}\left|x^{\prime}(s)\right| d s \leq D_{1}+\int_{0}^{T}\left|x^{\prime}(s)\right| d s .
$$

On the other hand, multiplying the two sides of $(3.18)[0, T]$, we get

$$
\int_{0}^{T} g\left(x\left(t-\tau_{2}(t)\right)\right) d t=\int_{0}^{T} e_{2}(t) d t
$$

and

$$
\int_{0}^{T} \frac{g(x(t))}{1-\tau_{2}^{\prime}\left(\gamma_{2}(t)\right)} d t=\int_{0}^{T} e_{2}(t) d t
$$

It follows from [A7] that

$$
\left|\int_{0}^{T} g(x(t)) d t\right|=\int_{0}^{T}|g(x(t))| \geq \sigma \int_{0}^{T}|x(t)|^{m} d t
$$

and so

$$
\min _{s \in[0, T]}\left|\frac{1}{1-\tau_{2}^{\prime}\left(\gamma_{2}(s)\right)}\right| \sigma \int_{0}^{T}\left|x^{\prime}(t)\right|^{m} d t \leq \int_{0}^{T}\left|e_{2}(t)\right| d t .
$$

It follows from (3.19), (3.20), and the Hölder inequality that there exists a positive constant $C_{1}$ such that

$$
\int_{0}^{T}\left|x^{\prime}(t)\right|^{m} d t \leq C_{1}
$$


and

$$
|x|_{0} \leq D_{1}+T^{1 / n} C_{1}^{1 / m}:=C_{2},
$$

where $\frac{1}{m}+\frac{1}{n}=1$. The rest of the proof of the theorem is identical to that of Theorem 3.1.

Remark 3.4 From the proof of the above theorems, we find that the prior bounds estimation to the periodic solutions is key for obtaining existence results. Here, we use a new method for obtaining the prior bounds, which is different from the methods of [26] and [16].

\section{Uniqueness results}

In this section, we will study the uniqueness of periodic solutions to equations (1.6) and (1.7).

Theorem 4.1 Suppose that $\tau_{1}(t)=\varepsilon_{1}\left(\varepsilon_{1}\right.$ is sufficiently small constant) and the following condition holds:

[B1] $\left(u_{1}-u_{2}\right)\left[g\left(u_{1}\right)-g\left(u_{2}\right)\right]<0$ for all $u_{1}, u_{2} \in \mathbb{R}, u_{1} \neq u_{2}$.

Then equation (1.6) has at most one T-periodic solution.

Proof Denote

$$
y(t)=\varphi_{p}\left(\frac{x^{\prime}(t)}{\sqrt{1+x^{\prime 2}(t)}}\right)+F(x(t))
$$

where $F(x(t))$ satisfies $\frac{d F(x(t))}{d t}=f\left(x^{\prime}(t)\right)$. Then (1.6) can be changed into the following form:

$$
\left\{\begin{aligned}
x^{\prime}(t) & =\frac{\varphi_{q}(y(t)-F(x(t)))}{\sqrt{1-\varphi_{q}^{2}(y(t)-F(x(t)))}}, \\
y^{\prime}(t) & =g\left(x\left(t-\varepsilon_{1}\right)\right)+e_{1}(t) .
\end{aligned}\right.
$$

The rest of the proof of the theorem is identical to that of Theorem 4.1 in [17].

Theorem 4.2 Suppose that $\tau_{1}(t)=\varepsilon_{1}$ ( $\varepsilon_{1}$ is sufficiently small constant), assumptions [A1], [A3], [A5], and [B1] hold, then equation (1.6) has a unique T-periodic solution $x$.

Theorem 4.3 Suppose that $\tau_{1}(t)=\varepsilon_{1}$ ( $\varepsilon_{1}$ is a sufficiently small constant), and assumptions [A2], [A4], [A5], and [B1] hold, then equation (1.6) has a unique T-periodic solution $x$, if

(1) $m=p, \sigma>\max _{t \in[0, T]}\left|\frac{1}{1-\tau_{1}^{\prime}\left(\gamma_{1}(t)\right)}\right| r$, or

(2) $m<p$.

We also have the following uniqueness results to (1.7).

Theorem 4.4 Suppose that $\tau_{2}(t)=\varepsilon_{2}$ ( $\varepsilon_{2}$ is sufficiently small constant) and assumption [B1] hold. Then equation (1.7) has at most one T-periodic solution $x$.

Theorem 4.5 Suppose that $\tau_{2}(t)=\varepsilon_{2}$ ( $\varepsilon_{2}$ is sufficiently small constant), assumptions [A6], [A7], and [B1] hold, then equation (1.7) has a unique T-periodic solution $x$. 
Remark 4.1 We give an example for verifying the existence of $F(x(t))$. Let $F(x(t))=$ $5 x(t), f\left(x^{\prime}(t)\right)=5 x^{\prime}(t)$. Then we have $\frac{d F(x(t))}{d t}=5 x^{\prime}(t)=f\left(x^{\prime}(t)\right)$.

Remark 4.2 In [16], based on the delay $\tau(t)=0$, Wang gave uniqueness results to equation (1.6), our results allow $\tau(t)$ to be a small positive constant, and this is more significant than the work of Wang.

\section{Examples}

As applications, we provide some examples to illustrate the main results.

\section{Example 5.1}

$$
\left(\varphi_{4}\left(\frac{x^{\prime}(t)}{\sqrt{1+x^{\prime 2}(t)}}\right)\right)^{\prime}+\left(x^{\prime}\right)^{3} e^{\sin x^{\prime}}+g\left(x\left(t-\frac{1}{2} \sin t\right)\right)=\cos t
$$

where $p=4, f\left(x^{\prime}\right)=\left(x^{\prime}\right)^{3} e^{\sin x^{\prime}}, \tau(t)=\frac{1}{2} \sin t, e(t)=\cos t, T=2 \pi$,

$$
g(u)= \begin{cases}-e^{(2+\cos u)} & \text { for } u \geq 0 \\ -\frac{1}{2} u & \text { for } u<0\end{cases}
$$

Conclusion Equation (5.1) has at least one $2 \pi$-periodic solution.

Proof From $f\left(x^{\prime}\right) x^{\prime}=\left(x^{\prime}\right)^{4} e^{\sin x^{\prime}}$ and $\sigma\left|x^{\prime}\right|^{4}$, choose $\sigma<e^{-1}$, then assumption [A1] holds. From $f(0)+|e|_{0}=1$, choose $D=2$, then assumption [A3] holds. From $g^{\prime}(u)$ being bounded, then assumption [A5] holds. Applying Theorem 3.1, equation (5.1) has at least one $2 \pi$ periodic solution.

\section{Example 5.2}

$$
\left(\varphi_{4}\left(\frac{x^{\prime}(t)}{\sqrt{1+x^{\prime 2}(t)}}\right)\right)^{\prime}+\left(x^{\prime}\right)^{4}+g\left(x\left(t-\frac{1}{2} \sin t\right)\right)=\cos t
$$

where $p=4, f\left(x^{\prime}\right)=\left(x^{\prime}\right)^{4}, \tau(t)=\frac{1}{2} \sin t, e(t)=\cos t, T=2 \pi$,

$$
g(u)= \begin{cases}-u e^{\sin u} & \text { for } u \geq 0 \\ -\frac{1}{2} u & \text { for } u<0\end{cases}
$$

Conclusion Equation (5.2) has at least one $2 \pi$-periodic solution.

Proof Let $m=p=4,0<\sigma \leq 1$. We have

$$
f\left(x^{\prime}\right)=\left(x^{\prime}\right)^{4} \geq \sigma\left|x^{\prime}\right|^{4}, \quad \lim _{|x| \rightarrow+\infty} \frac{|g(x)|}{|x|^{p-1}}=0:=r
$$

and

$$
\sigma>\max _{t \in[0, T]}\left|\frac{1}{1-\tau_{1}^{\prime}\left(\gamma_{1}(t)\right)}\right| r=0
$$


Thus, all conditions of Theorem 3.2 hold. Applying Theorem 3.2, equation (5.2) has at least one $2 \pi$-periodic solution.

\section{Example 5.3}

$$
\left(\varphi_{4}\left(\frac{x^{\prime}(t)}{\sqrt{1+x^{\prime 2}(t)}}\right)\right)^{\prime}+g\left(x\left(t-\frac{1}{2} \sin t\right)\right)=\cos t
$$

where $p=4, \tau(t)=\frac{1}{2} \sin t, e(t)=\cos t, T=2 \pi$,

$$
g(u)= \begin{cases}-e^{(2+\cos u)} & \text { for } u \geq 0 \\ -\frac{1}{2} u & \text { for } u<0\end{cases}
$$

Conclusion Equation (5.3) has at least one $2 \pi$-periodic solution.

Proof From $|e|_{0}=1$, choose $D_{1}=2$, then assumption [A6] holds. If $u<0$, choose $0<\sigma \leq \frac{1}{2}$ and $m=1$, then assumption [A7] holds. If $u \geq 0$, choose $\sigma$ sufficiently small and $m=1$, then assumption [A7] also holds. Applying Theorem 3.3, equation (5.3) has at least one $2 \pi$-periodic solution.

\section{Conclusions}

In this paper, we have investigated a periodic solution problem for two types of $p$-Laplacian prescribed mean curvature equations. Some sufficient conditions are obtained for the existence and uniqueness of periodic solutions to the above equations. Three examples are given to show the effectiveness of the results in this paper.

We mention here that the technique used here is different from the usual methods employed to solve periodic solutions cases such as the Mawhin continuation theorem and some fixed point theorems. We develop a new method (i.e., a generalized Mawhin continuation theorem) for $p$-Laplacian equations. This method is more extensively applicable than classical methods.

Competing interests

The authors declare that they have no competing interests.

Authors' contributions

All authors contributed equally to the writing of this paper. All authors read and approved the final manuscript.

\section{Author details}

'Department of Mathematics, Huaiyin Normal University, Huaian, Jiangsu 223300, P.R. China. ${ }^{2}$ Department of Mathematics, Beijing Institute of Technology, Beijing, 100081, P.R. China.

\section{Acknowledgements}

This project was funded by NNSF (No. 11571136) of China.

Received: 21 May 2016 Accepted: 4 October 2016 Published online: 22 October 2016

\footnotetext{
References

1. Hale, J: Theory of Functional Differential Equations. Applied Math. Sciences, vol. 3. Springer, Berlin (1977)

2. Wang, G: A priori bounds for periodic solutions of delay Rayleigh equation. Appl. Math. Lett. 12, 41-44 (1999)

3. Lu, S, Chen, L: The problem of existence of periodic solutions for neutral functional differential system with nonlinear difference operator. J. Math. Anal. Appl. 387, 1127-1136 (2012)

4. Zhang, M: Periodic solutions of linear and quasilinear neutral functional differential equations. J. Math. Anal. Appl. 189, 378-392 (1995)

5. Lu, S, Ren, J, Ge, W: Problems of periodic solutions for a kind of second order neutral functional differential equation. Appl. Anal. 82, 411-426 (2003)
} 
6. Du, B, Guo, L, Ge, W, Lu, S: Periodic solutions for generalized Liénard neutral equation with variable parameter. Nonlinear Anal. TMA 70, 2387-2394 (2008)

7. Zhu, Y, Lu, S: Periodic solutions for $p$-Laplacian neutral functional differential equation with deviating arguments. J. Math. Anal. Appl. 325, 377-385 (2007)

8. Zhu, Y, Lu, S: Periodic solutions for $p$-Laplacian neutral functional differential equation with multiple deviating arguments. J. Math. Anal. Appl. 336, 1357-1367 (2007)

9. Liu, B, Yu, J: On the existence of solutions for the periodic boundary value problems with $p$-Laplacian operator. J. Syst. Sci. Math. Sci. 23, 76-85 (2003)

10. Cheung, W, Ren, J: On the existence of periodic solutions for $p$-Laplacian generalized Liénard equation. Nonlinear Anal. 60, 65-75 (2005)

11. Cheung, W, Ren, J: Periodic solutions for p-Laplacian Rayleigh equations. Nonlinear Anal. 65, 2003-2012 (2006)

12. Lu, S, Ge, W: Sufficient conditions for the existence of periodic solutions to some second order differential equations with a deviating argument. J. Math. Anal. Appl. 308, 393-419 (2005)

13. Lu, S, Zhong, T, Chen, L: Periodic solutions for $p$-Laplacian Rayleigh equations with singularities. Bound. Value Probl. 2016, 96 (2016)

14. Aizicovici, S, Papageorgiou, NS, Staicu, V: Periodic solutions for second order differential inclusions with the scalar p-Laplacian. J. Math. Anal. Appl. 322, 913-929 (2006)

15. Peng, S, Zhu, S: Periodic solutions for P-Laplacian Rayleigh equations with a deviating argument. Nonlinear Anal. 67 , 138-146 (2007)

16. Wang, D: Existence and uniqueness of periodic solutins for prescribed mean curvature Rayleigh type $p$-Laplacian equation. J. Appl. Math. Comput. 46, 181-200 (2014)

17. Li, Z, An, T, Ge, W: Existence of periodic solutions for a prescribed mean curvature Liénard $p$-Laplacian equation with two delays. Adv. Differ. Equ. 2014, 290 (2014)

18. Ge, W, Ren, J: An extension of Mawhin's continuation theorem and its application to boundary value problems with a p-Laplacian. Nonlinear Anal. 58, 477-488 (2004)

19. Wang, L, Shao, J: New results of periodic solutions for a kind of forced Rayleigh-type equations. Nonlinear Anal., Real World Appl. 11, 99-105 (2010)

20. Li, Y, Huang, L: New results of periodic solutions for forced Rayleigh-type equations. J. Comput. Appl. Math. 231, 99-105 (2008)

21. Ding, T, lannacci, R, Zanolin, F: Existence and multiplicity results for periodic solutions of semilinear Duffing equations. J. Differ. Equ. 105, 364-409 (1993)

22. Ma, T: Periodic solutions of Rayleigh equation via time-maps. Nonlinear Anal. 75, 4137-4144 (2012)

23. Bonheure, D, Habets, P, Obersnel, F, Omari, P: Classical and non-classical solutions of a prescribed curvature equation. J. Differ. Equ. 243, 208-237 (2007)

24. Obersnel, F, Omari, P: Positive solutions of the Dirichlet problem for the prescribed mean curvature equation. J. Differ. Equ. 249, 1674-1725 (2010)

25. Benevieria, P, do Ó, JM, Medeiros, E: Periodic solutions for nonlinear systems with mean curvature-like operators. Nonlinear Anal. 65, 1462-1475 (2006)

26. Feng, M: Periodic solutions for prescribed mean curvature Liénard equation with a deviating argument. Nonlinear Anal., Real World Appl. 13, 1216-1223 (2012)

27. Pino, M, Guerra, I: Ground states of a prescribed mean curvature equation. J. Differ. Equ. 241, 112-129 (2007)

28. Gaines, R, Mawhin, J: Coincidence Degree and Nonlinear Differential Equations. Springer, Berlin (1977)

29. Yoshizawa, T: Stability Theory by Lyapunov' Second Methods. Math. Society of Japan, Tokyo (1966)

30. Massera, J: The existence of periodic solutions of systems of differential equations. Duke Math. J. 17, 457-475 (1950)

\section{Submit your manuscript to a SpringerOpen ${ }^{\circ}$ journal and benefit from:}

- Convenient online submission

Rigorous peer review

- Immediate publication on acceptance

Open access: articles freely available online

- High visibility within the field

- Retaining the copyright to your article 